\title{
Introducing Memristor to Electrical and Electronic Engineering Undergraduates
}

\author{
Wang Shibing ${ }^{1,2, a}$, Yang Yashuai ${ }^{1, b}$, Wang Jing ${ }^{1, c}$ \\ ${ }^{1}$ School of Computer and Information Engineering, Fuyang Normal University, China \\ ${ }^{2}$ Faculty of Electronic Information and Electrical Engineering, Dalian University of Technology, China \\ awang_shibing@dlut.edu.cn, b1339983887@qq.com, ${ }^{\mathrm{c}} 710813573 @ q q . c o m$
}

Keywords: Memristor, Electrical \& Electronic Engineering, Undergraduates.

\begin{abstract}
Memristor is considered as the fourth fundamental circuit element along with resistor, capacitor, and inductor. Due to its features of non-volatility, nonlinearity, low power consumption, it has enormously potential applications in electrical \& electronic science and engineering fields, and has become a popular research directions. So, it is time to introduce and teach memristor to the undergraduates of electrical \& electronic engineering (EEE) for broadening their horizons of memristor and laying a foundation for their future work in related fields. In this paper, the necessity and feasibility of introducing memristor to EEE undergraduates are analyzed firstly. Then the relevant teaching objectives and contents, teaching modes and methods are explored and discussed. Finally, some conclusions are drawn that introducing memristor to EEE undergraduates is necessary, while the teaching contents should be open and optional, and the teaching modes and methods can be flexible.
\end{abstract}

\section{Introduction}

In 1971, Leon Chua at the University of California (Berkeley) proposed a new two-terminal circuit element named memristor and its practical active circuit model [1]. He supposed that memristor might had been fabricated but was improperly identified, and predicted that physical memristors could be invented. However, nobody had discovered or invented physical memristors for 37 years. Until 2008, R. Stanley Williams at HP Labs announced that they found the missing memristor and presented a physical model of memristor (i.e., Titanium dioxide $\left(\mathrm{TiO}_{2}\right)$ thin film memristor) [2]. Since then, some new memristor porotypes (e.g. polymeric memristor, resonant tunneling memristor, spintronic memristor, spin-transfer torque memristor, etc.) were invented successively [3], and memristors attracted more and more attentions from the academic and industrial communities in the field of physics, EEE, materials, computer science, nanoscience and nontechnology, etc. The research achievements show that the memristors have widely potential applications in programmable analog circuits and digital gate arrays, nonlinear circuits and systems, computer memory, neural networks [4-7].

Since memristor is so important in the field of EEE, should we introduce and teach memristor to EEE undergraduates? Some scientists and teachers of EEE have discussed this problem enthusiastically, and most of them agreed to introduce memristor to EEE undergraduates [8-13]. Leon Chua insisted that memristor should be included in electronics textbooks as a standard topic [8]. Giovanni E. Pazienza etc. considered that the memristor should have placed in the electrical engineering curricula even before than in the labs [9]. Frank Y. Wang maintained that the concept of memristor was exceedingly useful and should be introduced to students as early as possible [10]. However, in the actual teaching, the vast majority of teachers do not teach memristor to EEE undergraduates for the reasons that the memristors stay in the laboratory research stage currently and must be analyzed by nonlinear methods.

In our opinion, it is necessary and feasibility to introduce memristor to EEE undergraduate as early as possible, but it is not necessary to revise the textbooks, to limit teaching contents, and to restrict teaching modes and methods at present. In this paper, we will explore and discuss this topic from 
three aspects including the necessity, contents, and methods of introducing memristor to EEE undergraduates.

\section{Why Should We Introduce Memristor to EEE Undergraduates?}

Why should we introduce memristor to EEE undergraduates? The major reasons include two aspects: one is the necessity of introducing memristor to EEE graduates from the perspective of theory and application, the other is the feasibility of introducing memristor to EEE undergraduates from the view of research achievements.

As shown in Fig. 1, it is widely known that there are four circuit variables, namely, current (i), voltage $(v)$, charge $(q)$, and magnetic flux $(\varphi)$, which might be used six mathematical equations to connect pairs of these four variables. Leon Chua deduced the existence of memristor to fill up the missing link between charge and magnetic flux. In his opinion, memristor is not an invention but a discovery, and memristive phenomena will appear in Nano-electronics circuits ubiquitously [1, 8]. So, from the theoretic point of view, the EEE undergraduates need to accept the complete and accurate circuit principle with memristor.

Memristor, being a fundamental circuit element, is inevitably used to design circuits and systems, such as electrical filters, programmable analog circuits, nonlinear circuits and systems (e.g., the simplest chaotic circuit as shown in Fig. 2 [14]), which can generate chaotic or hyperchaotic sequences for digital image encryption and secure communication. Due to its non-volatile memory function, memristor is most potentially applied to non-volatile random access memory and programmable crossbar arrays, hybrid digital circuits, which may change Von Neumann's computer architecture in the future. Furthermore, memristor can change its resistance nonlinearly by the current passing through it, so it is properly used to design synaptic circuits in artificial neural networks. To sum up, memristor has tremendously potential applications in electrical and information fields, we must introduce and teach memristor to EEE undergraduates in order to train the future engineers who need to be familiar with memristor and to be flexible enough to incorporate latest developments [8].

In recent years, the academic researches and technology developments of memristors, have made fruitful achievements. Until Oct. 28, 2015, there are 822 article-type papers in Web of Science with the search query “TITLE: (memristor) OR TITLE: (memristive)", in which the category of EEE owns $34.185 \%$ (281) and holds the first place. These papers have reported the research progress of the memristors and their applications in the field of EEE, including various physical models, mathematical models, and equivalent circuit models of the memristors, generation and analysis of memristor-based circuits and systems, etc. Such research results and online resources can provide abundant materials for introducing memristor to EEE undergraduates.

\section{Why Do We Introduce Memristor to EEE Undergraduates?}

What should we introduce to EEE undergraduates? There is no fixed and standard answer for this problem in different universities and majors. However, the main contents include concept and applications of memristor.

Concept of Memristor. The emergence of memristor updates and further perfects the basic theory of circuit. EEE undergraduates need to be familiar with the definition, types, characteristics, and models of memristor. Memristor is a two-terminal passive fundamental electronic component, which can be defined by the relationship between charge and flux-linkage, i.e., $f(\varphi, q)=0$. If this relationship is a single-valued function of charge $q$ (as shown in Fig. 3(a)), the memristor is called charge-controlled memristor or current-controlled memristor. If the function $\mathrm{f}$ is a single-valued function of flux $\varphi$, the memristor belongs to flux-controlled memristor or voltage-controlled memristor. Eq.(1) and Eq.(2) describe these two types of memristors respectively.

$$
M(q)=\frac{d \varphi(q)}{d q}, v(t)=M(q) i(t), \quad i=\frac{d q(t)}{d t}
$$




$$
W(\varphi)=\frac{d q(\varphi)}{d \varphi}, \quad i(t)=W(\varphi) v(t), \quad v=\frac{d \varphi(t)}{d t}
$$

where $M(q)$ and $W(\varphi)$ denote memristance and memductance which are nonlinear functions of $q$ and $\varphi$ respectively. So, the Voltage-Current Relation (VCR) of memristor is nonlinear, and pinched hysteresis loop exists as shown in Fig. 3(b).

Recently, some new memristive porotypes have been reported, but such physical device models are difficult for EEE undergraduates except the major of microelectronics. Nevertheless, the simplified mathematical models are much simpler for EEE undergraduates, and piece-wise linear function, smooth continuous cubic nonlinear function, Chebyshev polynomials, etc. have been used to describe the memristors, e.g. reference [14] proposed a charge-controlled memristor model described by Eq. (3). Furthermore, the equivalent circuit models are usually used to simulate in PSpice and Multisim, and to perform the circuit experiments.

$$
\begin{aligned}
& V_{\mathrm{M}}=\beta\left(x^{2}-1\right) i_{\mathrm{M}} \\
& \&=i_{\mathrm{M}}-\alpha x-i_{\mathrm{M}} x
\end{aligned}
$$

Applications of Memristor. As mentioned in section 2, memristor has extensively potential applications in nonlinear circuits and systems, non-volatile memory, artificial neural networks, etc. It is impossible to introduce all of the applications to EEE undergraduates. Therefore, different applications should be selected for EEE undergraduates from different specialties in order to broaden their vision, for example, memristor-based crossbar latches for microelectronic specialty, memristor-based nonlinear circuits and systems for electrical \& electronic specialty, memristor-based neural networks for control and computer specialty.

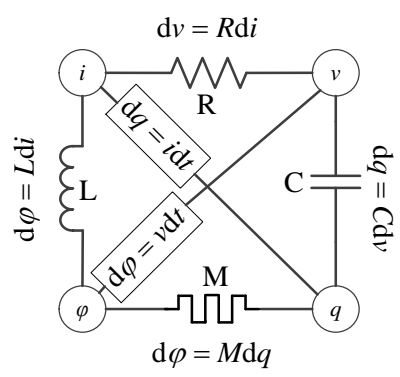

Figure 1. The four fundamental circuit elements

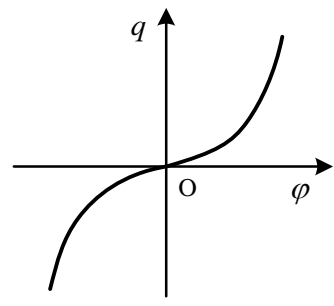

(a) Charge-controlled memristor

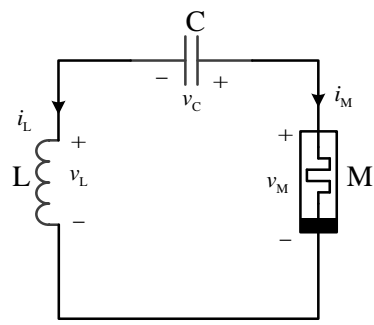

Figure 2. Memristor-based chaotic circuit

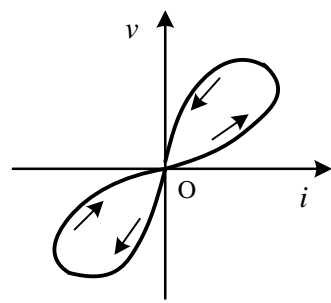

(b) Current-voltage characteristics

Figure 3. $I-V$ and $\varphi-q$ characteristics of a charge-controlled memristor 


\section{How Do We Introduce Memristor to EEE Undergraduates?}

How to introduce memristor to EEE undergraduates? In this section, we will present three teaching modes for answering and discussing this problem.

Course-Embedded Mode. Up to now, memristor has not been written into the textbooks, also not listed in the compulsory contents for EEE undergraduates. So the most feasible approach is to embed the contents into the electrical and electronic courses, such as circuit principle, analog electronics, digital electronics, automatic control, artificial intelligence, and so on, as shown in Fig. 4. When the teacher of an EE course teaches the relevant contents, he can introduce memristor and its applications to his class by means of theoretical instruction, simulation demonstration, or guiding the students to self-exploration.

It is worth mentioning that simulation demonstration is considered as the most effective teaching method currently. Leon Chua has pointed out that memristor circuits would likely be taught initially by computer simulations, without knowing much about nonlinear theories and techniques. For example, we can construct the state functions of the simplest chaotic memristor-based circuit (as shown in Fig. 2) based on Kirchhoff's voltage law (KVL) and Kirchhoff's current law (KCL), and achieve Eq. (4) by assuming $x=v_{C}, y=i_{L}[14]$.

$$
\left\{\begin{array}{l}
\&=\frac{1}{C} y \\
\&=-\frac{1}{L}\left[x+\beta\left(z^{2}-1\right) y\right] \\
\&=-y-\alpha z+y z
\end{array}\right.
$$

Then we can construct Simulink model, or edit Matlab codes for numerical simulation. Finally, we set $\alpha=0.6, \beta=1.5, L=3, C=1,1.132,1.3,1.4$ respectively, and simulate the model, resulting in phase portraits and time-domain waveforms of different $C$ as shown in Fig. 5, which indicate that the circuit can operate in various periodic states and chaotic state under different circuit parameters. Thus it can be seen that only simple circuit theory and basic programing ability are needed to achieve visual description of the memristor-based circuits and systems.

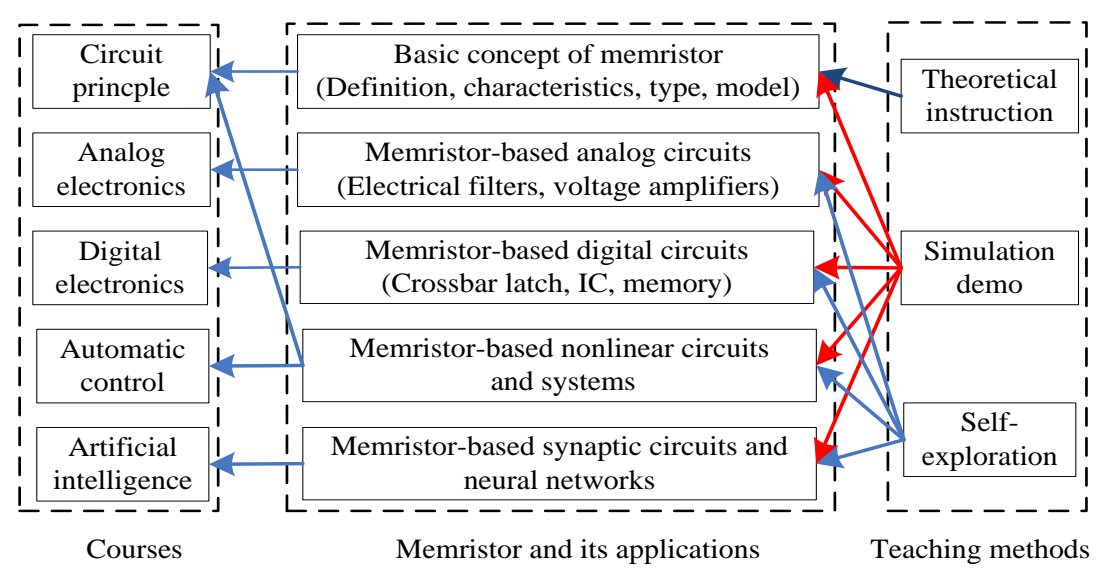

Figure 4. Course-embedded mode

Extracurricular Activities Mode. In addition to the course-embedded mode, we can also guide the EEE undergraduates, who have interests and abilities to study memristor ulteriorly, to participate in extracurricular scientific and technological activities. The teachers may invite the students to join their memristor research group, encourage the students to apply for the undergraduate innovative projects, and guide the students to choose memristor as the topic of graduation theses. In our university, some undergraduate students have approved National Undergraduate Training Programs 
for Innovation and Entrepreneurship of China with the title of Simulation and analysis of memristor-based chaotic circuits and systems, Digital image encryption based on memristive chaotic and hyperchaotic systems, etc., based on which, some students have published their achievements and obtained Chinese software copyrights, the others accomplished their graduation theses.

Expert-Report Mode. The research progress of memristor is changing rapidly, and the teachers could not be familiar with everything about memristor. So, it is feasible and effective to invite the experts of memristor, including scientists, scholars, engineers and technologists, to make special reports on memristor and its applications, which are beneficial to widen the students' view of memristor, as well as to stimulate the teachers’ ideas of researching memristor.
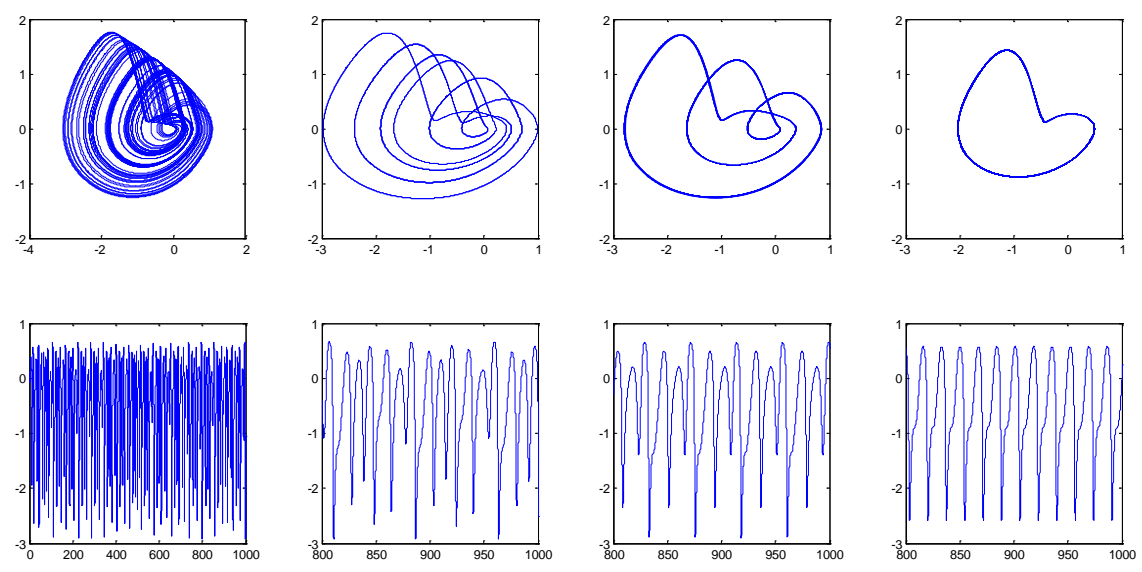

Figure 5. Dynamics of the simplest memristor-based chaotic circuit

\section{Conclusions}

Memristor, being the fourth fundamental electronic component, has enriched the circuit elements, and improved the circuit theory. Due to its characteristics of non-volatility, nonlinearity, nanoscale, easily integrated, and low power consumption, memristor has been shown vastly potential applications in EEE fields, which inevitably need EEE undergraduates to be familiar with memristor and its applications. So it is time to introduce and teach memristor to EEE undergraduates. The teachers should introduce the concept and partial applications of memristor to EEE undergraduates according to the different specialty requirements and the teaching situations, and train EEE undergraduates incorporating latest developments of memristor by adopting course-embedded mode, extracurricular activities mode, and expert-report mode flexibly.

\section{Acknowledgement}

This research was supported by the Natural Science Foundation of Anhui Provincial Universities (No. KJ2012A214), the Teaching Quality Project of Anhui Province (NO. 2013gxk063, NO.2012jyxm336, NO.2013jyxm132, NO. 2014jyxm224, NO.sjjd077), and National Undergraduate Training Programs for Innovation and Entrepreneurship of China (NO. 201410371020).

\section{References}

[1] L. O. Chua, Memristor - The missing circuit element. IEEE Transactions on Circuit Theory, vol. 18 (1971), p. 507-519.

[2] D. B. Strukov, G. S. Snider, D. R. Stewart, R. S. Williams, The missing memristor found, Nature, vol. 453 (2008), p. 80-83. 
[3] Wang L., Yang, C., Wen, J., Gai S., Peng Y X., Overview of emerging memristor families from resistive memristor to spintronic memristor. Journal of Materials Science-Materials in Electronics, vol. 26 (2015), p. 4618-4628.

[4] D.Sacchetto, G. De Micheli, Y. Leblebici, Multiterminal Memristive Nanowire Devices for Logic and Memory Applications: A Review. Procedings of the IEEE, vol. 100 (2012), p. 2008-2020.

[5] Y. Chen, G. Liu, C. Wang, W. Zhang, RW. Li, L. Wang, Polymer memristor for information storage and neuromorphic applications. Materials Horizons, vol. 1 (2014), p. 489-506.

[6] M. Itoh, L. O. Chua, Memristor Oscillators. International Journal of Bifurcation and Chaos, vol. 18 (2008), p. 3183-3206

[7] A. Thomas, Memristor-based neural networks. Journal of Physics D-Applied Physics, vol. 46 (2013), Article ID. 093001.

[8] S. Adee, How do you teach the memristor? (2010), 20 October 2015, http://spectrum.ieee.org/ tech-talk/semiconductors/nanotechnology/do-you-teach-the-memristor

[9] G. E.Pazienza, J. Albo-Canals, Teaching memristors to EE undergraduate Students. IEEE Circuits and Systems Magazine, 11 (2011) 36-44.

[10] R. C. Johnson, “Missing link” memristor created: Rewrite the textbooks? (2008), 20 October 2015, http://www. eetimes.com/document.asp?doc_id=1168454

[11] F. Y. Wang, Memristor for introductory physics (2008), 20 October 2015, http://arxiv.org/abs /0808.0286

[12] M. J. Kumar, Memristor - why do we have to know about it? IETE Technical Review, vol. 26 (2009), p. 3-6.

[13] B. Eleonora, B. Eugenia, P. Pietro, Chaos at school: Chua's circuit for students in junior and senior high school. International Journal of Bifurcation and Chaos, vol. 20 (2010), p. 1-28.

[14] B. Muthuswamy, L. O. Chua, Simplest chaotic circuit. International Journal of Bifurcation and Chaos, vol. 20 (2010), p. 1567-1580. 